Document downloaded from:

http://hdl.handle.net/10251/176989

This paper must be cited as:

Blasco-Brusola, A.; Navarrete-Miguel, M.; Giussani, A.; Roca-Sanjuan, D.; Vayá Pérez, I.; Miranda Alonso, MÁ. (2020). Regiochemical memory in the adiabatic photolysis of thyminederived oxetanes. A combined ultrafast spectroscopic and CASSCF/CASPT2 computational study. Physical Chemistry Chemical Physics. 22(35):20037-20042.

https://doi.org/10.1039/d0cp03084h

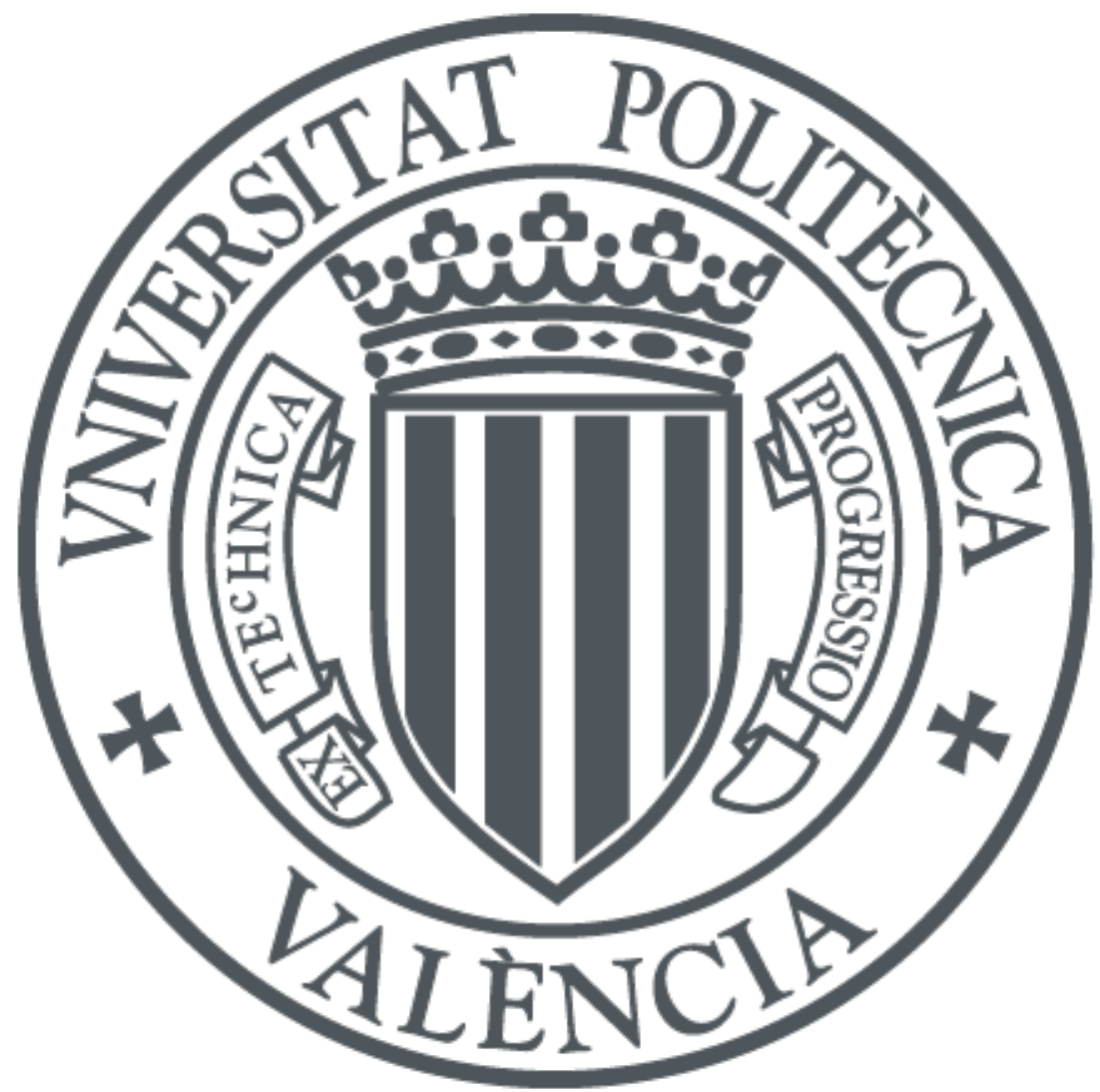

The final publication is available at

https://doi.org/10.1039/d0cp03084h

Copyright The Royal Society of Chemistry

Additional Information 
Received 00th January 20xx, Accepted 00th January 20xx DOI: $10.1039 / \times 0 \times x 00000 x$

\section{Regiochemical memory in the adiabatic photolysis of thymine- derived oxetanes. A combined ultrafast spectroscopic and CASSCF/CASPT2 computational study}

\author{
Alejandro Blasco-Brusola, a: Miriam Navarrete-Miguel, b* Angelo Giussani, ${ }^{\mathrm{b}}$ Daniel Roca-Sanjuán, ${ }^{* b}$ \\ Ignacio Vayá, ${ }^{* a}$ and Miguel A. Miranda*a
}

\begin{abstract}
The photoinduced cycloreversion of oxetanes has been thoroughly investigated in connection with the photorepair of the well-known DNA (6-4) photoproducts. In the present work, the direct photolysis of the two regioisomers arising from irradiation of benzophenone (BP) and 1,3-dimethylthymine (DMT), namely the head-to-head (HH-1) and head-to-tail (HT-1) oxetane adducts, has been investigated by combining ultrafast spectroscopy and theoretical multiconfigurational quantum chemistry analysis. Both experimental and computational results agree with the involvement of an excited triplet exciplex ${ }^{3}[\mathrm{BP} \cdots \mathrm{DMT}]^{*}$ for the photoinduced oxetane cleavage to generate ${ }^{3} \mathrm{BP} *$ and DMT through an adiabatic photochemical reaction. The experimental signature of ${ }^{3}[\mathrm{BP} . . \mathrm{DMT}]^{*}$ is the appearance of an absorption band at $c a .400 \mathrm{~nm}$, detected by femtosecond transient absorption spectroscopy. Its formation is markedly regioselective, as it is more efficient and proceeds faster for $\mathrm{HH}-1$ ( $\sim 2.8 \mathrm{ps}$ ) than for HT-1 ( $6.3 \mathrm{ps})$. This is in line with the theoretical analysis, which predicts an energy barrier to reach the triplet exciplex for HT-1, by contrast with a less hindered profile for $\mathrm{HH}-1$. Finally, the more favorable adiabatic cycloreversion of HH-1 compared to HT-1 is explained by its lower probability to reach the intersystem crossing with the ground state, which would induce a radiationless deactivation process leading either to the starting adduct or to the dissociated BP and DMT.
\end{abstract}

\section{Introduction}

Solar ultraviolet light is known to induce cellular DNA damage through a number of processes including the dimerization of neighboring pyrimidines. ${ }^{1-4}$ Cyclobutane pyrimidine dimers (CPDs) and to a lesser extent pyrimidine (6-4) pyrimidone photoproducts ((6-4)PPs) are the most abundant UV-induced lesions in DNA; ${ }^{4-6}$ their formation may result in the appearance of mutations, cell death and cancer. $^{7-9}$ In humans, these lesions are normally repaired through a process known as nucleotide excision repair; however, other organisms (i.e. bacteria, plants, etc.) have developed efficient photorepair mechanisms ${ }^{10-13}$ that involve binding of a specific enzyme to the DNA lesion and reactivation through a photoinduced electron transfer process. ${ }^{14-21}$

Previously, the photoinduced cycloreversion of oxetanes generated from 1,3-dimethylthymine (DMT) and carbonyl compounds has been investigated as a model for the photoenzymatic repair of DNA (64)PPs. ${ }^{22-30}$ Direct photolysis of some oxetane derivatives was

\footnotetext{
a. Departamento de Química/Instituto de Tecnología Química UPV-CSIC, Universitat Politècnica de València, Camino de Vera $s / n, 46022$ València (Spain).

b. Instituto de Ciencia Molecular, Universitat de València, P.O. Box 22085, 46071 València (Spain).

†Electronic Supplementary Information (ESI) available: Experimental details, spectroscopic and theoretical data. See DOI: 10.1039/x0xx00000x

†These authors contributed equally to get the experimental and theoretical data, respectively.
}

observed to result in a rare adiabatic cleavage leading to the triplet excited state of the corresponding carbonyl species, along with ground state DMT.24, 30 A more recent work has proposed that cycloreversion takes place in a stepwise manner from the singlet excited state, with population of the triplet excited carbonyl at a later stage. ${ }^{28}$ All these studies have been performed on the chemically stable head-to-head $(\mathrm{HH})$ oxetane obtained from the Paternò-Büchi reaction between DMT and the photoactive carbonyl derivative (this is also the regioisomer structurally closer to the purported intermediate formed between two adjacent thymines that ultimately leads to the (6-4)PP); however, the photobehavior of its chemically less stable head-to-tail ( $\mathrm{HT}$ ) regioisomer, which is also obtained in the reaction, ${ }^{31,32}$ has not yet been addressed.

With this background, in order to gain deeper insight into the mechanism of the photoinduced cycloreversion of thymine-derived oxetanes, the photobehavior of the head-to-head and head-to-tail regioisomers obtained from benzophenone (BP) and DMT ( $\mathrm{HH}-1$ and HT-1, respectively; see Scheme 1) has been investigated by means of a combined experimental and theoretical approach. This involves transient absorption spectroscopy from the femtosecond to the microsecond time scales, in addition to a detailed computational analysis including multiconfigurational quantum-chemistry calculations (complete-active-space self-consistent field/completeactive-space second-order perturbation theory, CASSCF/CASPT2). Thus, ultrafast spectroscopy in tandem with high-level computational analysis have allowed us to settle the primary 
processes preceding the photolytic reactions with the aim of delineating the complex excited state relaxation processes that are involved.

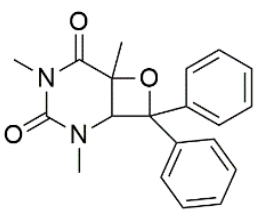

$\mathrm{HH}-1$

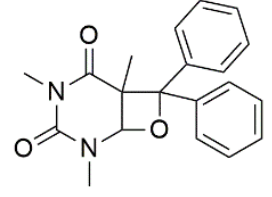

HT-1
Scheme 1 Chemical structures of head-to-head (HH-1) and head-to-tail (HT-1) DMT-BP oxetane regioisomers.

\section{Results and discussion}

The synthesis of HH-1 and HT-1 has been described earlier. ${ }^{31}$ Briefly, a solution containing DMT $(100 \mathrm{mM})$ and BP $(200 \mathrm{mM})$ in acetonitrile (MeCN) was placed into Pyrex tubes and irradiated $c a$. 8h under nitrogen in a multilamp Luzchem photoreactor emitting at $\lambda_{\max }=350$ $\mathrm{nm}(12 \times 8 \mathrm{~W}$ lamps $)$. The crude was purified by column chromatography $\left(\mathrm{SiO}_{2}\right.$, hexane/ethyl acetate, $80 / 20$, v/v) followed by recrystallization of the separated fractions; the purity of both compounds was checked by ${ }^{1} \mathrm{H}-\mathrm{NMR}$ (see Fig. S1 in ESI). Direct steady-state UVC-photolysis of HH-1 and HT-1 gave rise to BP and DMT as the only photoproducts.

Laser flash photolysis (LFP) measurements were conducted in deaerated $\mathrm{MeCN}$ ( $\lambda_{\text {exc }}=266 \mathrm{~nm}$ ) on isoabsorptive solutions at the excitation wavelength for $\mathrm{HH}-1, \mathrm{HT}-1$ and $\mathrm{BP}$, as a reference compound. Since oxetanes can in principle be partially degraded during the measurements, fresh solutions were employed after each light pulse, in order to rule out possible artifacts due to secondary excitation of photodegradation products.

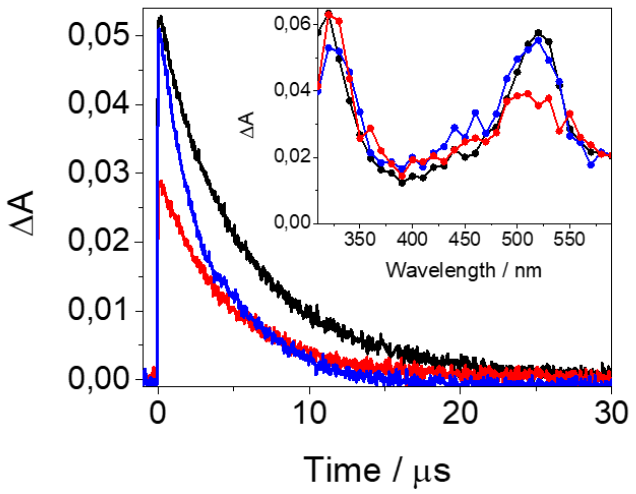

Fig. 1 LFP decay traces for BP (black), HH-1 (blue) and HT-1 (red) at $530 \mathrm{~nm}$ upon excitation at $266 \mathrm{~nm}$ in deaerated MeCN. The inset shows the transient absorption spectra $150 \mathrm{~ns}$ after the laser pulse.

The profile of the transient absorption spectra for HH-1 and HT-1 was very similar to that of ${ }^{3} \mathrm{BP} *$ (inset in Fig. 1). ${ }^{33}$ However, a weak absorption in the $350-400 \mathrm{~nm}$ region was noticed for both regioisomers. The most interesting finding was the observation of a transient absorption at $530 \mathrm{~nm}$ (where ${ }^{3} \mathrm{BP} *$ displays its maximum) ${ }^{34}$ immediately after the laser pulse. Our results indicate that, within the duration of the shot, a practically quantitative adiabatic population of the triplet excited state of benzophenone is indeed accomplished for $\mathrm{HH}-1 .{ }^{24}$ By contrast, photolysis of $\mathrm{HT}-1$ also induced population of ${ }^{3} \mathrm{BP} *$ albeit to a much lesser extent ( $c a .60 \%$ ). Thus, a clear regiodifferentiation has been observed in the adiabatic photochemical reaction, which was much more effective for $\mathrm{HH}-1$. The decay traces followed a one order law with lifetimes of about 5 $\mu$ s for BP and HT-1 and slightly shorter for $\mathrm{HH}-1$ ( $3.5 \mu \mathrm{s})$.

The weak absorption in the 350-400 $\mathrm{nm}$ region detected for both oxetanes could be associated with a transient species with some ${ }^{3} \mathrm{DMT}^{*}$ character, as the maximum of the triplet-triplet absorption of thymine (Thy) is centered at $370 \mathrm{~nm} .{ }^{35,} 36$ In this context, LFP measurements ( $\lambda_{\text {exc }}=355 \mathrm{~nm}$ ) were conducted in deaerated MeCN on mixtures containing BP and increasing amounts of DMT; under these conditions, the only absorbing species is BP. As shown in Fig. $2 \mathrm{~A}$, a clear quenching of ${ }^{3} \mathrm{BP} *$ was evidenced upon addition of $\mathrm{DMT}$, with a rate constant of $c a .1 .2 \times 10^{9} \mathrm{M}^{-1} \mathrm{~s}^{-1}$, which is in the order of the previously reported value. ${ }^{23}$
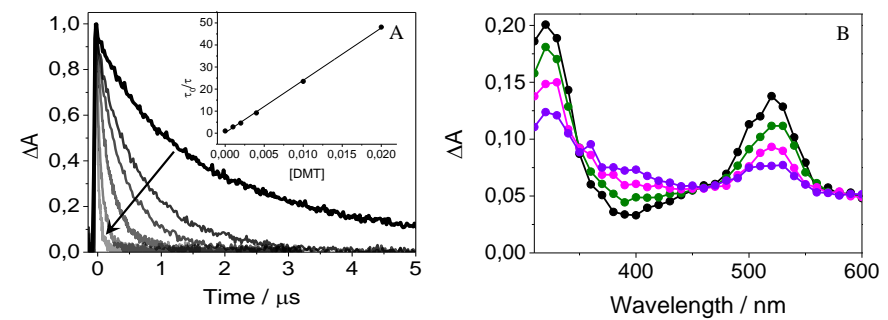

Fig. 2 (A) Normalized LFP decay traces for BP/DMT mixtures (from 1:0 to 1:10) at $530 \mathrm{~nm}$. The inset shows the Stern-Volmer analysis for ${ }^{3} \mathrm{BP} *$ quenching. (B) LFP absorption spectra for BP (black) and BP/DMT mixtures at 1:1 (green), 1:5 (magenta) and 1:10 (violet) molar ratios recorded $30 \mathrm{~ns}$ after the laser pulse. Measurements were performed upon excitation at $355 \mathrm{~nm}$ in deaerated MeCN.

The nature of ${ }^{3} \mathrm{BP} *$ quenching upon interaction with thymine derivatives has been discussed previously, and attributed mainly to the Paternò-Büchi reaction. ${ }^{23}$, 26, 37 Oxetane formation has been proposed to proceed through 1,4-diradical intermediates of triplet nature, ${ }^{23}$ and recently the involvement of triplet exciplex precursors ${ }^{3}[\mathrm{BP} \text {...Thy }]^{*}$ has also been inferred. ${ }^{37}$ This is in fact not surprising in view of the number of examples dealing with oxetane formation through complex-type intermediates such as exciplexes. ${ }^{38}$ In fact, the absorption spectra of Fig. 2B clearly show the appearance of a new absorption around $400 \mathrm{~nm}$ that increases with DMT concentration. A similar transient has been detected in related products and assigned to ${ }^{3}$ Thy*; 37 however, a triplet exciplex-like state between BP and DMT ([BP...DMT]*) cannot be discarded. Indeed, this species would be expected to contain the signatures of both BP and DMT triplet excited states, as it is actually the case for the detected transient. In order to investigate the possible participation of the triplet exciplex ${ }^{3}[\mathrm{BP} \ldots \mathrm{DMT}]^{*}$ in the cycloreversion processes, a computational analysis based on multiconfigurational quantumchemistry and the CASSCF/CASPT2 method efficiently combined with density functional theory (DFT) has been performed for $\mathrm{HH}-1$ and $\mathrm{HT}$ 1. Computational details as well as analyses to benchmark the methodology can be found in ESI. This high-level analysis allowed us to achieve an interpretation of the experimental observations, and 
to obtain clear evidence supporting the chemical mechanism involved in the photoinduced oxetane cleavage (see Fig. 3).
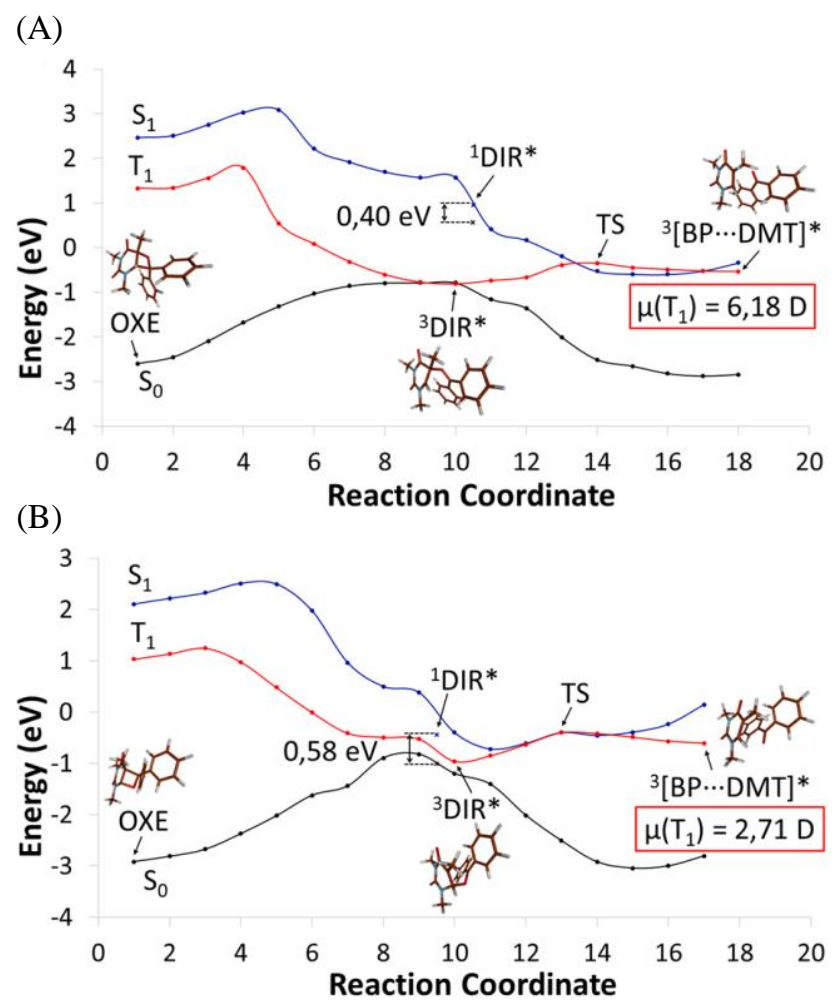

Fig. 3 Chemical mechanism of the photoinduced cycloreversion process for $\mathrm{HH}-1$ (A) and HT-1 (B). Double-headed arrows indicate the energy gap between $S_{0}$ and $S_{1}$ at the $S_{1}$ equilibrium structure in the diradical region $\left({ }^{1} \mathrm{DIR}^{*}\right)$. The reference energy is the sum of the separated triplet BP and ground state DMT molecules. The reaction coordinate, according to the optimized structures, is mostly associated with the breaking of the CC and $\mathrm{CO}$ bonds between DMT and BP. See Tables S1-S5 for further details on the electronic-structure and methodological aspects and Tables S6-S7 for benchmarking analyses in ESI.

Five key equilibrium structures characterize the photoinduced cycloreversion process: the ground state $\left(S_{0}\right)$ oxetane (OXE), the singlet $\left(\mathrm{S}_{1}\right)$ and triplet $\left(\mathrm{T}_{1}\right)$ diradicals $\left({ }^{1} \mathrm{DIR} \mathrm{R}^{*}\right.$ and ${ }^{3} \mathrm{DIR} \mathrm{R}^{*}$, respectively) with the $\mathrm{C}-\mathrm{C}$ bond broken, the triplet exciplex ('[BP...DMT]*) and the transition state (TS) between ${ }^{3} \mathrm{DIR}^{*}$ and ${ }^{3}[\mathrm{BP} \cdots \mathrm{DMT}]^{*}$ related to the $\mathrm{C}-\mathrm{O}$ bond cleavage. Irradiation of the oxetanes induces population of the excited singlet electronic state. As can be seen from left to right in Fig. 3, the energy of $\mathrm{S}_{1}$ decreases significantly along the $\mathrm{C}-\mathrm{C}$ bond breaking step towards the region of the diradical (DIR*; point 10 in Fig. 3). At this region, an important difference between the two regioisomers can be observed. The energy gap $\mathrm{S}_{1}-\mathrm{S}_{0}$ for HT-1 $(0.80$ $\mathrm{eV})$ is much lower than that for $\mathrm{HH}-1(2.35 \mathrm{eV})$ applying the same methodological procedure for determining the properties of the diradical structure. Such trend is maintained by comparing the statespecific (SS) and multistate (MS) approaches of the CASPT2 (Table S7 in ESI), which according to previous studies, ${ }^{39}$ points to a solid conclusion. The distinct behavior can be attributed to the different nature of $S_{1}$ at this region, as it can be observed in the electrondensity difference between $S_{0}$ and $S_{1}$ for each regioisomer (see Fig. 4). Thus, $S_{1}$ in HT-1 develops a charge transfer character from DMT to BP (see also the large dipole moment in Table S1 in ESI), typical of the oxetane ring electronic structure; similar features were previously detected for the thymine-thymine dimer formation. ${ }^{40}$ Meanwhile, $\mathrm{S}_{1}$ in $\mathrm{HH}-1$ displays a high multiconfigurational character with contributions from electronic configurations corresponding to excitations delocalized over BP and the diradical configuration localized at the broken bond (see Table S2 in ESI). This coupling between electronic configurations increases the energy splitting between $S_{0}$ and $S_{1}$.
(A)

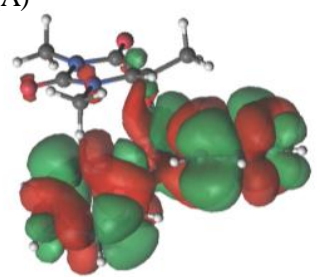

(B)

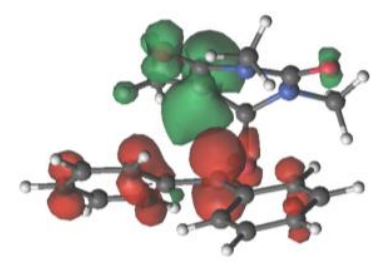

Fig. $4 \mathrm{~S}_{1}-\mathrm{S}_{0}$ electron-density difference at the ${ }^{3} \mathrm{DIR}$ * geometry for (A) HH-1 and (B) HT-1, computed from the CASSCF wavefunctions of each electronic state. Green and red colors indicate departure and arrival regions, respectively, of electron-density in the $S_{0}$ to $S_{1}$ excitation. See the nature of the orbitals contributing to the electron densities in Figs. S3 and $\mathrm{S} 8$ in ESI.

Computational searches of the $S_{1}$ minimum $\left({ }^{1} D I R^{*}\right)$ at the diradical region (with only the $\mathrm{C}-\mathrm{C}$ bond broken) decrease the energy gap between $\mathrm{S}_{0}$ and $\mathrm{S}_{1}$ down to 0.58 and $0.40 \mathrm{eV}$ for $\mathrm{HT}-1$ and $\mathrm{HH}-1$, respectively. From this point, the system can access a nearby conical intersection ( $\mathrm{Cl}$ ) (not computed here but estimated in the surroundings) to decay non-radiatively to the ground state or to continue the $\mathrm{S}_{1}$ surface towards the dissociation of the $\mathrm{C}-\mathrm{O}$ bond of the oxetane ring. The latter process is favorable considering the downhill energy profile towards the singlet-triplet crossing (STC) with the $T_{1}$ surface. This STC involving the energy profiles of $S_{1}$ and $T_{1}$ is responsible of the so-called "adiabatic" generation of "BP* upon excitation of HH-1 or HT-1, which occurs via a triplet exciplex. Nonnegligible spin-orbit coupling (SOC) values have been determined for both regioisomers $\left(\sim 4 \mathrm{~cm}^{-1}\right)$ at the points where $S_{1}$ and $T_{1}$ become near-degenerate, which allows population of the triplet state. However, significant differences appear for HH-1 and HT-1 regarding the probability of this "adiabatic" $S_{1} \rightarrow T_{1}$ path. Thus, for HH-1, $S_{1}$ crosses $\mathrm{T}_{1}$ at the TS region related to the $\mathrm{C}-\mathrm{O}$ bond breaking on the triplet $\mathrm{T}_{1}$ manifold (see Fig. $3 \mathrm{~A}$ ), while for $\mathrm{HT}-1$ this crossing occurs before the TS and becomes trapped at the diradical region due to the energy barrier to the transition state (Fig. 3B). This is a consequence of the large $\mathrm{S}_{0}-\mathrm{S}_{1}$ gap at the diradical and the fact that ${ }^{1} \mathrm{DIR}$ * is much more energetic for HH-1 than for HT-1. In the latter, ${ }^{1}{ }^{D I R}{ }^{*}$ almost coincides energetically with the equilibrium geometry of the triplet diradical ( $\left.{ }^{3} \mathrm{DIR}^{*}\right)$

At the ${ }^{3} \mathrm{DIR}$ * diradical, the ground state $\mathrm{S}_{0}$ and the lowest-lying triplet excited state $\mathrm{T}_{1}$ show a small energy gap for both $\mathrm{HH}-1$ and $\mathrm{HT}-1$, and therefore this point corresponds to a singlet-triplet crossing. According to the wavefunctions (see Tables S1 and S2 in ESI), both $\mathrm{S}_{0}$ and $T_{1}$ states have a diradical nature (with the unpaired electrons at the $C$ atoms of the broken bond and non-parallel and parallel spins for $S_{0}$ and $T_{1}$, respectively). This STC point, if reached, shall allow non- 
radiative decay via intersystem crossing (ISC), even though the SOC, lower than $0.5 \mathrm{~cm}^{-1}$ in both systems, points to a slow process.

Therefore, the fact that $\mathrm{HH}-1$ has a high ${ }^{1} \mathrm{DIR}$ * energy, clearly above the energy of ${ }^{3}[\mathrm{BP} \cdots \mathrm{DMT}]^{*}$, in addition to the $\mathrm{S}_{1} / \mathrm{T}_{1} \mathrm{STC}$ occurring at the TS, points to a favorable production of the triplet exciplex. On the contrary, for HT-1, the lower ${ }^{1} \mathrm{DIR}^{*}$ energy and the earlier $\mathrm{S}_{1} / \mathrm{T}_{1} \mathrm{STC}$ nearby the $S_{0} / T_{1}$ STC in addition to the energy barrier to reach TS, makes the evolution towards ${ }^{3}[\mathrm{BP}$...DMT]* less competitive as compared to the non-radiative decays to the ground state. These findings agree with the lower intensity of the transient absorption band at $530 \mathrm{~nm}$ detected by LFP for HT-1.

In order to obtain further key mechanistic information on the early stages of oxetane cycloreversion, and in an attempt to provide additional experimental evidence supporting the formation of triplet-like excimer states, the photobehavior of both regioisomers was investigated by means of femtosecond transient absorption spectroscopy and further computational analysis on ${ }^{3}[\mathrm{BP} \cdots \mathrm{DMT}]^{*}$. Previous reports have shown that excitation of BP at $360 \mathrm{~nm}$ induces formation of a broad transient band from 500 to $600 \mathrm{~nm}$ with maximum at $575 \mathrm{~nm}$, assigned to its singlet-singlet absorption band. The intersystem crossing to the triplet excited state, with maximum centered at $525 \mathrm{~nm}$, has been found to proceed within ca. 9.6 ps. ${ }^{41}$ This is in good agreement with a later work performed at $\lambda_{\text {exc }}=267$ $\mathrm{nm}$, where deactivation of the benzophenone singlet excited state at $340 \mathrm{~nm}$, leading to the triplet through ISC, has been found to occur in about 10.6 ps. $^{28}$ Herein, isoabsorptive solutions of $\mathrm{HH}-1, \mathrm{HT}-1$ and $\mathrm{BP}$ in MeCN were subjected to femtosecond laser excitation at 280 $\mathrm{nm}$, making sure that the degree of photodegradation was lower than $5 \%$ at the end of each experiment. The transient absorption spectra from 0.1 to 40 ps are shown in Fig. S14 in ESI. In the case of $\mathrm{BP}$, an absorption band with maximum at $530 \mathrm{~nm}$ was formed within ca. $10.4 \mathrm{ps}$, along with the deactivation of the band at $340 \mathrm{~nm}$, associated to ${ }^{1} \mathrm{BP} *$ (Figs. 5A and $\mathrm{D}$ ); this value for the ISC is very similar to those previously reported. ${ }^{28,41}$
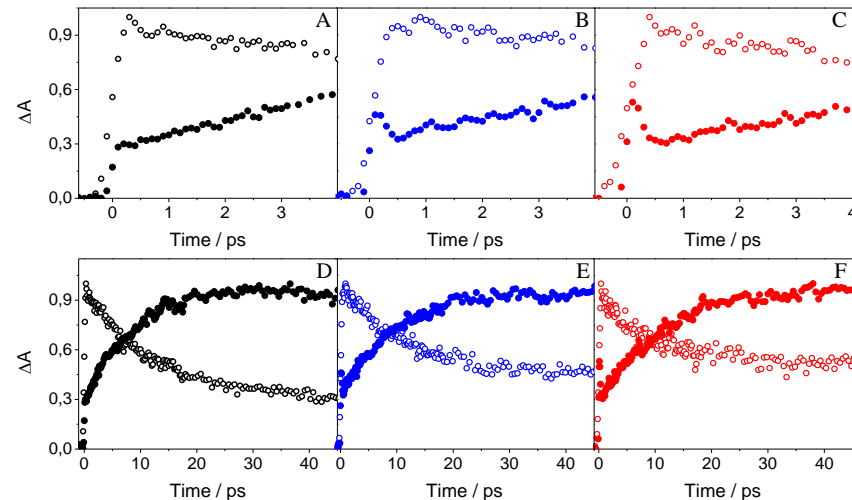

Fig. 5 Femtosecond kinetic traces at $340 \mathrm{~nm}$ (open circles) and $530 \mathrm{~nm}$ (solid circles) at different time windows for: (A) and (D) BP; (B) and (E) HH-1 and (C) and (F) HT-1 after excitation at $280 \mathrm{~nm}$ in MeCN.

Analysis of the kinetics at $530 \mathrm{~nm}$, where the singlet excited state also exhibits some absorption very early after excitation, ${ }^{41}$ revealed an ultrafast decay of $\sim 270 \mathrm{fs}$ for both HH-1 and HT-1 (Figs. 5B and C), not detected for BP. This time constant, which is in the order of that reported by Kwok et al., ${ }^{28}$ was attributed to the C-C bond scission from the singlet state to generate a diradical intermediate. After the initial ultrafast decay, the trace at $530 \mathrm{~nm}$ started to grow, in accordance with the triplet excited state formation, with a lifetime of $\sim 10.7 \mathrm{ps}$, which is very similar to that detected for BP.
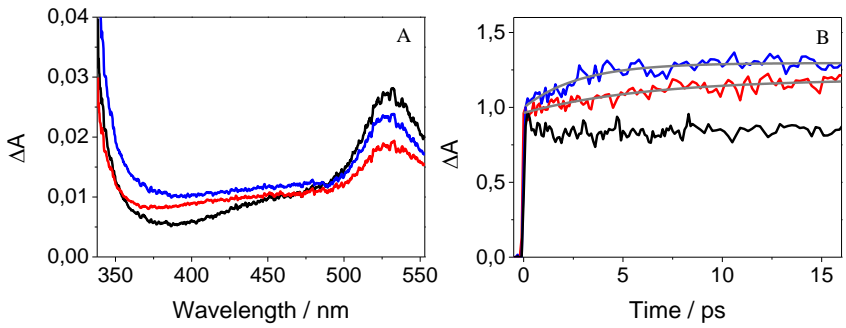

Fig. 6 (A) Femtosecond transient absorption spectra recorded 20 ps after the laser pulse, and (B) normalized kinetic traces at $400 \mathrm{~nm}$ for BP (black), HH-1 (blue) and HT-1 (red) upon excitation at $280 \mathrm{~nm}$ in $\mathrm{MeCN}$. The best exponential fit for the data is shown in gray.

It is worth to highlight the absorption detected around $400 \mathrm{~nm}$ for both regioisomers (Fig. 6A), which is absent in BP and lower for HT-1 than for $\mathrm{HH}-1$; as stated above, this band can be taken as a signature of the triplet exciplex state ${ }^{3}[\mathrm{BP} \ldots \mathrm{DMT}]^{*}$, which is supported by computational analysis. Thus, significant binding energies were obtained for ${ }^{3}[\mathrm{BP} . . . \mathrm{DMT}]^{*}$ of around $10-12 \mathrm{kcal} / \mathrm{mol}$ for both regioisomers (see Table S3 in ESI), which points to relatively stable triplet excited state structures. Such binding interactions are due to the delocalization of the excitation among both BP and DMT that characterizes the wavefunction of ${ }^{3}[\mathrm{BP} \ldots \mathrm{DMT}]^{*}$ (see Tables S1 and S2 in ESI). Note that the exciplex maintains the $\pi \pi^{*}$ nature of the BP moiety upon increasing the accuracy of the CASPT2 methodology (Table S7 in ESI).

The triplet exciplex displayed a slightly higher binding energy ( 2 $\mathrm{kcal} / \mathrm{mol}$ ) for $\mathrm{HH}-1$ than for $\mathrm{HT}-1$ (see Table S3 in ESI), which is related to the higher dipole moment found for $\mathrm{HH}-1$ (see Fig. 3). Experiments using solvents with lower dielectric constant than MeCN were attempted to verify whether the exciplex signature at $c a .400 \mathrm{~nm}$ changes the relative intensity between $\mathrm{HH}-1$ and HT-1 since computations predict an inversion of stability in non-polar solvents (see Table S3 in ESI). However, hydrogen transfer from the benzophenone triplet leading to the ketyl radical was detected, which prevented reproducing the same types of measurements and analyses as in MeCN.

An additional piece of evidence supporting the formation of triplet exciplexes was provided by the computed transient absorption spectra for the more stable $\mathrm{HH}-1$ exciplex (Tables S4 and S5 in ESI). Herein, it was identified a strong transition $\left(T_{1} \rightarrow T_{7}\right)$ occurring at 378 $\mathrm{nm}$, which can be directly related to the absorption detected experimentally at $c a .400 \mathrm{~nm}$. As we computed in previous studies, ${ }^{42}$ $\mathrm{T}_{1} \rightarrow \mathrm{T}_{\mathrm{n}}$ and $\mathrm{T}_{2} \rightarrow \mathrm{T}_{\mathrm{n}}$ transitions characteristic of isolated ${ }^{3} \mathrm{BP} *$ occur at energies out of the $400 \mathrm{~nm}$ region, and therefore ${ }^{3} \mathrm{BP} *$ cannot be responsible for the absorption observed at this wavelength.

Further analysis of the kinetics at $400 \mathrm{~nm}$ (Fig. 6B) evidenced a flat profile for BP; by contrast, both oxetane regioisomers (specially $\mathrm{HH}$ 1) displayed a clear growth, in agreement with ${ }^{3}[\mathrm{BP} \cdots \mathrm{DMT}]^{*}$ formation, which was faster for HH-1 ( 2.8 ps) than for HT-1 ( 6.3 
ps). These results are in full agreement with those obtained from computational analysis. Thus, the faster and more efficient formation of the transient at $\sim 400 \mathrm{~nm}$ for HH-1 (compared to HT-1) is directly related to its lower energy barrier to reach the triplet exciplex. This is also in line with the higher degree of adiabatic cycloreversion observed for $\mathrm{HH}-1$, as in this case it is much less likely to reach the $\mathrm{Cl}$ where radiationless deactivation leads back to the starting oxetane or to the dissociated BP and DMT in the ground state.

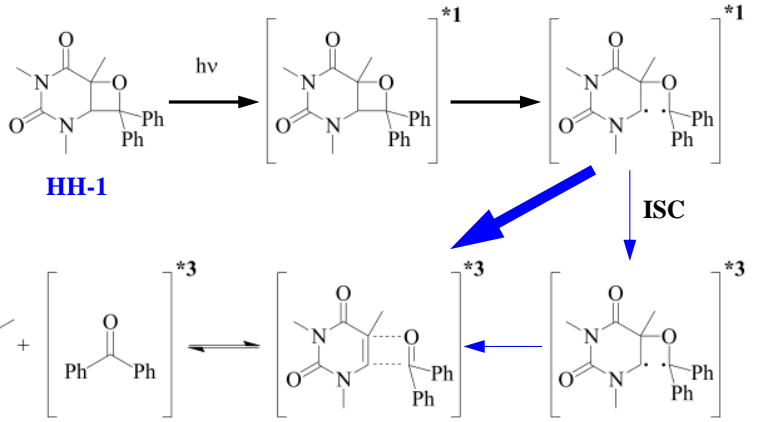

DMT ${ }^{3} \mathbf{B P} *$ ${ }^{3}[\mathrm{BP} \cdots \mathrm{DMT}]^{*}$ $(\sim \mathbf{1 0 0 \% )}$

(B)
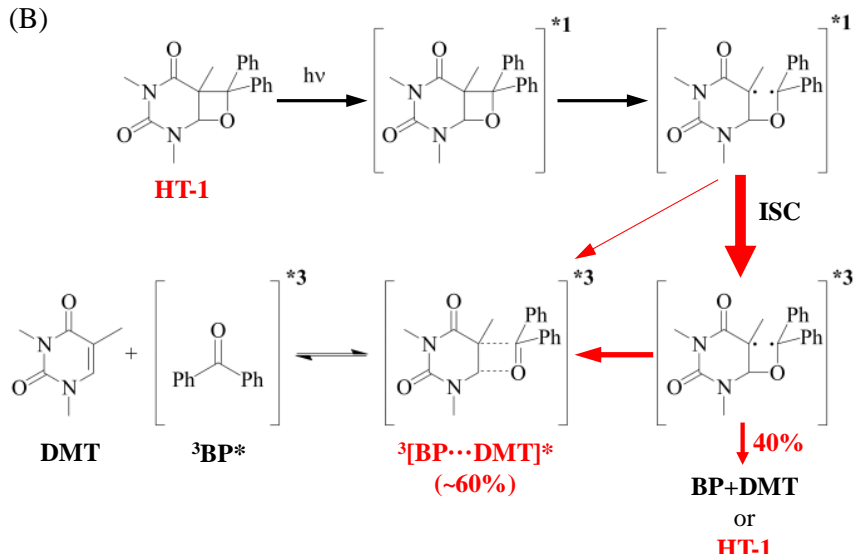

Scheme 2 Proposed mechanism for the photoinduced cycloreversion process for $\mathrm{HH}-1$ (A) and HT-1 (B). The width of the colored arrows represents the efficiency of a process.

In view of the photophysical and theoretical results, a detailed mechanism for the oxetane cycloreversion is proposed (Scheme 2). Note that this mechanism is different from the enzymatic repair, which is triggered by electron transfer. Direct photolysis of $\mathrm{HH}-1$ or HT-1 $\left(\lambda_{\text {exc }}=280 \mathrm{~nm}\right)$ instantaneously generates its excited singlet state. Then, diradical singlet intermediates are formed after $\sim 270 \mathrm{fs}$ through $\mathrm{C}-\mathrm{C}$ bond scission. For HT-1, the singlet diradicals cross efficiently to triplet diradical states, whose spectroscopic detection is difficult due to their low absorption around $530 \mathrm{~nm}$ and their spectral overlap with the benzophenone triplets. ${ }^{43-45}$ Then, around $40 \%$ are deactivated to the ground state by ISC while the other $60 \%$ evolve to exciplex-like states ${ }^{3}[\mathrm{BP} \ldots \mathrm{DMT}]^{*}$ in $c a .6 .3 \mathrm{ps}$. On the contrary, a highly-efficient ( $100 \%)$ and faster ( $2.8 \mathrm{ps})$ evolution from the singlet diradical to ${ }^{3}[\mathrm{BP} \cdots \mathrm{DMT}]^{*}$ occurs for $\mathrm{HH}-1$. The exciplexes present a spectral fingerprint characterized by an absorption at $\sim 400 \mathrm{~nm}$ and are in equilibrium with the dissociated ${ }^{3} \mathrm{BP} *(\sim 10.7 \mathrm{ps})$ and DMT.

\section{Conclusions}

The photoinduced cycloreversion of oxetanes derived from DMT and BP has been investigated by means of ultrafast spectroscopy and high-level computational analysis (CASSCF/CASPT2). When the photobehavior of both $\mathrm{HH}-1$ and HT-1 is compared, an interesting regiochemical memory is observed, revealed by significant differences related to the intermediates generated during oxetane cleavage. Ultrafast spectroscopy shows the regioselective formation of a triplet exciplex ${ }^{3}[\mathrm{BP} \ldots \mathrm{DMT}] *$ from diradical intermediates, which occurs to a much higher extent for $\mathrm{HH}-1$. These species (diradicals and exciplexes) have been identified by theoretical analysis, whose results agree with the experimental data. The differences observed in the adiabatic cycloreversion reaction between both regioisomers can be satisfactorily explained on the bases of both the theoretical and experimental results, and a mechanistic proposal is summarized in Scheme 2.

\section{Conflicts of interest}

There are no conflicts to declare.

\section{Acknowledgements}

Financial support from the Spanish Government (RYC-2015-17737, CTQ2017-89416-R, RYC-2015-19234, CTQ2017-87054-C2-2-P, and MDM-2015-0538), from the Consellería d'Educació, Investigació, Cultura i Esport (PROMETEO/2017/075 and GRISOLÍAP/2017/005) and from the Universitat de València (postdoctoral grant within the "Atracció de Talent 2019" Program for A. G.) is gratefully acknowledged. This work was also supported by a 2019 Leonardo Grant for Researchers and Cultural Creators, BBVA Foundation. The Foundation takes no responsibility for the opinions, statements, and contents of this project, which are entirely the responsibility of its authors.

\section{References}

1. D. E. Brash and W. A. Haseltine, Nature, 1982, 298, 189192.

2. J. Cadet and P. Vigny, in Bioorganic Photochemistry, ed. H. Morrison, John Wiley \& Sons, New York, 1990, pp. 1-272.

3. G. P. Pfeifer, Y. H. You and A. Besaratinia, Mutat. Res., 2005, 571, 19-31.

4. J. Cadet, S. Mouret, J. L. Ravanat and T. Douki, Photochem. Photobiol., 2012, 88, 1048-1065.

5. A. J. Varghese and S. Y. Wang, Science, 1968, 160, 186-187.

6. A. B. Fraga-Timiraos, V. Lhiaubet-Vallet and M. A. Miranda, Angew. Chem. Int. Ed., 2016, 55, 6037-6040.

$7 . \quad$ D. L. Mitchell, Photochem. Photobiol., 1988, 48, 51-57.

8. J.-S. Taylor, Acc. Chem. Res., 1994, 27, 76-82.

9. S.-L. Yu and S.-K. Lee, Mol. Cell. Toxicol., 2017, 13, 21-28. 
10. L. O. Essen and T. Klar, Cell. Mol. Life Sci., 2006, 63, 12661277.

11. T. Todo, H. Ryo, K. Yamamoto, H. Toh, T. Inui, H. Ayaki, T. Nomura and M. Ikenaga, Science, 1996, 272, 109-112.

12. T. Todo, H. Takemori, H. Ryo, M. Ihara, T. Matsunaga, $\mathrm{O}$. Nikaido, K. Sato and T. Nomura, Nature, 1993, 361, 371374.

13. T. Todo, H. Tsuji, E. Otoshi, K. Hitomi, S.-T. Kim and M. Ikenaga, Mutat. Res., 1997, 384, 195-204.

14. S. Asgatay, C. Petermann, D. Harakat, D. Guillaume, J. S. Taylor and P. Clivio, J. Am. Chem. Soc., 2008, 130, 1261812619.

15. C. Aubert, M. H. Vos, P. Mathis, A. P. M. Eker and K. Brettel, Nature, 2000, 405, 586-590.

16. S.-T. Kim, K. Malhotra, C. A. Smith, J.-S. Taylor and A. Sancar, J. Biol. Chem., 1994, 269, 8535-8540.

17. J. Li, Z. Liu, C. Tan, X. Guo, L. Wang, A. Sancar and D. Zhong, Nature, 2010, 466, 887-890.

18. M. P. Scannell, D. J. Fenick, S.-R. Yeh and D. E. Falvey, J. Am. Chem. Soc., 1997, 119, 1971-1977.

19. R. P. Sinha and D. P. Hader, Photochem. Photobiol. Sci., 2002, 1, 225-236.

20. X. Zhao, J. Liu, D. D. Hsu, S. Zhao, J.-S. Taylor and A. Sancar, J. Biol. Chem., 1997, 272, 32580-32590.

21. X. Zhao and D. Mu, Histol. Histopathol., 1998, 13, 11791182.

22. G. Prakash and D. E. Falvey, J. Am. Chem. Soc., 1995, 117, 11375-11376.

23. A. Joseph, G. Prakash and D. E. Falvey, J. Am. Chem. Soc., 2000, 122, 11219-11225.

24. A. Joseph and D. E. Falvey, J. Am. Chem. Soc., 2001, 123, 3145-3146.

25. A. Joseph and D. E. Falvey, Photochem. Photobiol. Sci., 2002, 1, 632-635.

26. S. Encinas, N. Belmadoui, M. J. Climent, S. Gil and M. A. Miranda, Chem. Res. Toxicol., 2004, 17, 857-862.

27. J. Trzcionka, V. Lhiaubet-Vallet, C. Paris, N. Belmadoui, M. J. Climent and M. A. Miranda, ChemBioChem, 2007, 8, 402407.

28. W. M. Kwok, X. Guan, L. M. Chu, W. Tang and D. L. Phillips, J. Phys. Chem. B, 2008, 112, 11794-11797.

29 Q. H. Song, H. B. Wang, W. J. Tang, Q. X. Guo and S. Q. Yu, Org. Biomol. Chem., 2006, 4, 291-298.

30.

N. Belmadoui, S. Encinas, M. J. Climent, S. Gil and M. A. Miranda, Chem. Eur. J., 2006, 12, 553-561.

31. X.-M. Hei, Q.-H. Song, X.-B. Li, W.-J. Tang, H.-B. Wang and Q.-X. Guo, J. Org. Chem., 2005, 70, 2522-2527.

32. K. Nakatani, T. Yoshida and I. Saito, J. Am. Chem. Soc., 2002, 124, 2118-2119.

33. J. A. Bell and H. Linschitz, J. Am. Chem. Soc., 1963, 85, 528532.

34. S. L. Murov, I. Carmichael and G. L. Hug, Handbook of Photochemistry, Marcel Dekker, New York, 2nd edn., 1993.

35 I. G. Gut, P. D. Wood and R. W. Redmond, J. Am. Chem Soc., 1996, 118, 2366-2373.

36. Z.-H. Zuo, S.-D. Yao, J. Luo, W.-F. Wang, J.-S. Zhang and N.Y. Lin, J. Photochem. Photobiol. B, 1992, 15, 215-222.

37. P. Miró, M. Gómez-Mendoza, G. Sastre, M. C. Cuquerella, M. A. Miranda and M. L. Marín, Chem. Eur. J., 2019, 25, 7004-7011.

38. J. Mattay, Angew. Chem. Int. Ed. Engl., 1987, 26, 825-845.
39. L. Serrano-Andres, M. Merchan and R. Lindh, J. Chem. Phys., 2005, 122, 104107.

A. Giussani, L. Serrano-Andres, M. Merchan, D. RocaSanjuan and M. Garavelli, J. Phys. Chem. B, 2013, 117, 1999-2004.

41. N. Tamai, T. Asahi and H. Masuhara, Chem. Phys. Lett., 1992, 198, 413-418.

M. Marazzi, S. Mai, D. Roca-Sanjuan, M. G. Delcey, R. Lindh, L. Gonzalez and A. Monari, J. Phys. Chem. Lett., 2016, 7, 622-626.

43. M. D'Auria and R. Racioppi, Molecules, 2013, 18, 1138411428.

44. S. C. Freilich and K. S. Peters, J. Am. Chem. Soc., 1981, 103, 6255-6257.

Z. B. Yang, R. B. Zhang and L. A. Eriksson, Phys. Chem. Chem. Phys., 2011, 13, 8961-8966. 